\title{
Endogenous testosterone density is an independent predictor of pelvic lymph node invasion in high-risk prostate cancer: results in 201 consecutive patients treated with radical prostatectomy and extended pelvic lymph node dissection
}

\author{
Antonio Benito Porcaro ${ }^{1}$ (1) - Alessandro Tafuri ${ }^{1,2,3}$. Andrea Panunzio ${ }^{1}$ - Giovanni Mazzucato ${ }^{1}$ Clara Cerrato ${ }^{1}$. \\ Sebastian Gallina ${ }^{1} \cdot$ Alberto Bianchi $^{1} \cdot$ Riccardo Rizzetto $^{1} \cdot$ Nelia Amigoni $^{1}$ - Emanuele Serafin ${ }^{1}$. \\ Francesco Cianflone ${ }^{1}$ - Rossella Orlando ${ }^{1}$ - Ilaria Gentile ${ }^{1}$ - Filippo Migliorini ${ }^{1}$. Stefano Zecchini Antoniolli ${ }^{1}$. \\ Giacomo Di Filippo ${ }^{4}$ - Matteo Brunelli ${ }^{5}$. Vincenzo Pagliarulo ${ }^{2}$. Maria Angela Cerruto ${ }^{1}$ - Alessandro Antonelli ${ }^{1}$
}

Received: 4 November 2021 / Accepted: 29 December 2021 / Published online: 19 January 2022

(c) The Author(s) 2022

\begin{abstract}
Objective To evaluate the influence of endogenous testosterone density (ETD) on pelvic lymph node invasion (PLNI) in high risk (HR) prostate cancer (PCa) treated with radical prostatectomy (RP) and staged with extended pelvic lymph node dissection (ePLND).

Materials and methods ETD was evaluated as the ratio of endogenous testosterone (ET) on prostate volume (PV). HR-PCa was assessed according to the European Association of Urology (EAU) system. The association of ETD and other routinely clinical factors (BPC: percentage of biopsy positive cores; PSA: prostate specific antigen; ISUP: tumor grade system according to the International Society of Urologic Pathology; cT: tumor clinical stage) with the risk of PLNI was assessed by the logistic regression model.

Results Overall, 201 out of 805 patients (24.9\%) were classified HR and PLNI occurred in 42 subjects (20.9\%). On multivariate analysis, PLNI was independently predicted by BPC (OR 1.020; 95\% CI 1.006-1.035; $p=0.019$ ), ISUP $>3$ (OR $2.621 ; 95 \%$ CI $1.170-5.869 ; p=0.019$ ) and ETD (OR 0.932; 95\% CI 0.870-0.999; $p=0.045$ ). After categorizing continuous clinical predictors, the risk of PLNI was independently increased by ETD up to the median (OR 2.379; 95\% CI 1.134-4.991; $p=0.022$ ), $\mathrm{BPC}>50 \%$ (OR 3.125; 95\% CI 1.520-6.425; $p=0.002$ ) as well as by ISUP > 3 (OR 2.219; 95\% CI 1.031-4.776; $p=0.042$ ).
\end{abstract}

Conclusions As ETD measurements decreased, patients were more likely to have PLNI. In HR disease with PLNI, the influence of PCa on ETD should be addressed by higher level studies.

Keywords Prostate cancer · High risk prostate cancer · Radical prostatectomy · Extended pelvic lymph node dissection . Pelvic lymph node invasion · Endogenous testosterone $\cdot$ Prostate volume $\cdot$ Endogenous testosterone density $\cdot$ Prostate specific antigen $\cdot$ Prostate specific antigen density $\cdot$ Percentage of biopsy positive cores density $\cdot$ Tumor load density

Antonio Benito Porcaro, Alessandro Tafuri, and Andrea Panunzio have contributed equally to this manuscript.

Antonio Benito Porcaro

drporcaro@yahoo.com

1 Department of Urology, University of Verona, Azienda Ospedaliera Universitaria Integrata, Verona, Italy

2 Department of Urology, Vito Fazzi Hospital, Lecce, Italy

3 Department of Neuroscience, Imaging and Clinical Sciences,

G. D’Annunzio University, Chieti, Italy
4 Department of General and Hepatobiliary Surgery, Azienda Ospedaliera Universitaria Integrata, University of Verona, Piazzale Stefani 1-37126, Verona, Italy

5 Department of Pathology, University of Verona, Azienda Ospedaliera Universitaria Integrata, Verona, Italy 


\section{Introduction}

Prostate cancer (PCa) is the second most diagnosed tumor in the aging male and its management has become a pivotal health problem in developing countries [1, 2]. According to European Association of Urology (EAU) and National Comprehensive Cancer Network (NCCN) guidelines, three PCa categories can be identified as related to the risk of developing metastases $[1,2]$. Among these, the high risk (HR) class is the most controversial for both classification among international societies and treatment which may include, for localized disease, radical prostatectomy (RP) with extended pelvic lymph node dissection (ePLND), radiotherapy (RT) and androgen suppression therapy and or a combination of multimodality treatments [1, 2]. When surgery is opted, an accurate anatomical staging using extended template is a critical issue for the high risk of pelvic lymph node invasion (PLNI) [1, 2]. However, residual disease may occur in the prostatic fossa and/or loco regional and retroperitoneal lymph nodes explaining prostate specific antigen (PSA) persistence or recurrence after surgery is a non-complete LND has been performed [1-3].

PCa has been related to several risk factors including genetic, dietary, environmental, physical (obesity, metabolic syndrome) and hormonal (hypogonadism) factors, as well $[1,2]$. Endogenous testosterone (ET) is an important factor for evaluating prostate growing disorders including either benign prostatic hyperplasia (BPH) and $\mathrm{PCa}$, which may also coexist. Our group has greatly focused its attention of the role of ET in PCa, finding that it could be associated with several unfavorable prognostic factors [4-6]. In low risk $\mathrm{PCa}$, we have recently shown that ET density (ETD), defined as the ratio of ET on prostate volume (PV), was an independent predictor of the risk of high tumor load (TL), which associated with unfavorable disease in the surgical specimen [7]. In the present study, we wanted to address the hypothesis that ETD may relate to PLNI in HR disease treated with RP and ePLND.

\section{Materials and methods}

\section{Study population}

The study was approved by Institutional Review Board. Informed consent was obtained by all subjects. Data were collected prospectively but retrospectively evaluated. In a period ranging from November 2014 to December 2019, 805 consecutive PCa patients who were not under androgen blockade had ET (nmol/L) measured at our lab before surgery and the test was performed at least 1 month after biopsies between 8.00 and 8.30 a.m. by radioimmunoassay. PSA (ng/mL), age (years), body mass index (BMI; kg/ $\mathrm{m}^{2}$ ), PV (mL) and percentage of BPC, the ratio of positive and total taken cores (\%), were evaluated in each case. PV was calculated by TRUS standard methods. Biopsies performed elsewhere were assessed for number of cores taken, tumor grade and PV. In our Institution, the 14-core trans perineal technique was used [8]. In each case, the ratios of BPC, PSA and ET with PV were calculated, and relative densities indicated as BPCD $(\% / \mathrm{mL})$, PSAD (ng/ $\left.\mathrm{ml}^{\wedge} 2\right)$ and $\operatorname{ETD}(\mathrm{nmol} /(\mathrm{dL} \times \mathrm{mL}))$, respectively. Clinical staging was assessed by the 2017 version of the TNM system (8th edition) with clinical $\mathrm{T}$ stage only referring to DRE findings and patients were classified into risk classes, as recommended by EAU guidelines [1]. Surgery, which was delivered by robot-assisted (RARP) or open approach (ORP), was performed by experienced surgeons. Extended PLND was performed according to guidelines [1, 2]. Nodal packets were submitted in separate packages according to a standard anatomical template including external iliac, internal iliac plus obturator, Marcille's common iliac, and Cloquet's nodal stations, bilaterally [9, 10]. Specimens including prostate and dissected lymph nodes were placed into formalin and evaluated by the dedicated pathologist. Prostates were weighted and tumors were graded according to the ISUP system $[1,2]$. Tumor quantitation was assessed as TL, which was defined as percentage of prostate involved by cancer; specifically, our dedicated pathologist assessed tumor quantitation by visual estimation of all the glass slides after all microscopically identifiable foci of carcinoma have been circled with a marked pen, as considered by ISUP association [11]. TL density (TLD) was calculated as the ratio of TL on prostate weight (\%/gr). Surgical margins were stated positive when cancer invaded the inked surface of the specimen. Removed lymph nodes were assessed for number and cancer invasion. Surgical specimens were staged by the 2017 version of the TNM system (8th edition), accordingly $[1,2]$.

\section{Statistical methods}

Continuous variables were measured for means (standard deviation, SD) and medians (interquartile range, IQR). Categorical factors were assessed for frequencies (percentages). Associations of ETD with clinical and pathological factors including PLNI were tested by the linear regression model (univariate and multivariate analysis). Appropriate biplots were computed, accordingly. Associations of ETD and other clinical/pathological factors with the risk of PLNI were assessed by the logistic regression model (univariate and multivariate analysis); furthermore, the discriminant power of significant continuous predictors were assessed by 
receiver operating characteristic (ROC) curve analysis with relative area under the curve (AUC). The software used to run the analysis was IBM-SPSS version 26. All tests were two-sided with $p<0.05$ considered to indicate statistical significance.

\section{Results}

\section{Demographics of the patient population}

Overall, 201 out of 805 PCa patients (24.9\%) were classified as HR class by EAU system [1]. Accordingly, ISUP grade groups 1 to 3 were diagnosed in 87 cases $(43.3 \%)$ and grade 4 to 5 in 114 subjects $(56.7 \%)$. Tumors were clinically staged as cT1c in 90 cases (44.8\%) and cT2/3 in $111(55.2 \%)$; furthermore, suspected nodal involvement was assessed in 34 cases (16.9\%). Mean (SD) and median (IQR) PSA were 12.5 (12.6) $\mathrm{ng} / \mathrm{dL}$ and 7.5 (5.4-14.1) $\mathrm{ng} / \mathrm{mL}$, respectively. The median (IQR) percentage of BPC was 50 (26.5-70). According to the preoperative physical status system by American Society of Anesthesiologists (ASA), 19 patients were ASA I (9.5\%), 161 ASA II (80.1\%) and 21 ASA III (10.4\%), as well. Surgery was delivered by the robotic approach in 171 $(85.1 \%)$ patients. Further details are reported in Table 1. In the surgical specimen, ISUP grade group $<4$ was detected in 86 subjects (42.8\%), while ISUP $>3$ was present in 115 cases $(57.2 \%)$; as such, tumor upgrading occurred 27 out of 87 ISUP $<4$ patients ( $31 \%$ ), while tumor downgrading was assessed in 26 out of 114 ISUP $>3$ cases (22.8\%). Cancers extended beyond the prostate in 75 cases $(37.3 \%)$ with extracapsular extension in 51 subjects $(25.4 \%)$ and seminal vesicle invasion in 24 patients (11.9\%). Positive surgical margins were detected in 75 subjects (37.3\%). The median (IQR) of counted lymph was 26 (20-32). Overall, PLNI occurred in 42 patients $(20.9 \%)$.

\section{Associations of ETD with clinical and pathological features}

Associations of ETD with clinical and pathological parameters were investigated by linear regression methods and reported in supplementary Table S1. On univariate analysis, ETD related positively to ET, PSAD, BPCD, TLD but inversely to PV, PW and PLNI. The positive association between ETD and ET is shown in supplementary Figure S1: as ET levels increased, ETD values also increased, accordingly. No significant associations were found out with the other remaining factors. On multivariate analysis model I (without TLD), ETD associated positively with BPCD (regression coefficient, $b=3.260$; 95\% CI 2.325; 4.195; $p<0.0001)$ and inversely with PLNI $(b=-3.324$; $95 \%$ CI $-5.557 ;-1.092 ; p=0.004)$. On multivariate model II (excluding BPCD), ETD positively associated with PSAD $(b=3.790 ; 95 \%$ CI $0.795 ; 6.785 ; p=0.013)$ and TLD $(b=2.995 ; 95 \%$ CI $0.859 ; 5.130 ; p=0.006)$ but inversely with PLNI $(b=-4.023 ; 95 \%$ CI $-6.540 ;-1.507$; $p=0.002$ ). Supplementary Figure S2 illustrates the positive association of ETD with PSAD: as PSAD increased ETD incremented accordingly but lower ETD values were detected in tumors associating with PLNI. Supplementary Figure $\mathrm{S} 3$ shows the positive association of ETD with TLD: as TLD incremented ETD increased accordingly; however, tumors associating with PLNI showed lower mean levels of ETD when compared with cancers without, as well.

Associations of ETD with TLD among EAU risk categories were also reported in supplementary Table S3 and illustrated in supplementary Figure S4. In the overall patient population including 805 patients, ETD and TLD were highly correlated to each other (Pearson's correlation coefficient, $r=0.214 ; p<0.0001)$. As EAU risk categories increased, TLD increased, accordingly. ETD did not show any significant distribution among EAU risk classes; however, it was an independent predictor of TLD together with EAU risk classes, as well. As ETD increased, TLD increased but increments for higher for intermediate and high-risk classes when compared to the low-risk class. Furthermore, as shown by the model, for same values of TLD (as an example TLD =1), measurements of ETD were significantly lower for the high-risk class meaning lower ET levels thus addressing features of aggressive disease.

\section{Associations of ETD with of PLNI}

Associations of clinical and pathological factors with the risk of PLNI are reported in Table 2. On univariate analysis, the risk of PLNI was significantly predicted by PSA, BPC, ISUP $>3, \mathrm{cT}>1 \mathrm{c}$ as well as by ETD with associations being positive for the first four predictors and inverse for the latter; furthermore, PLNI was also significantly predicted by pathological factors including TLD, seminal vesicle invasion, positive surgical margins and number of dissected lymph nodes with all predictors showing positive association. Figure 1 illustrates the inverse association between ETD and risk of PLNI. As depicted, significantly lower median levels of ETD were detected in tumors associated with PLNI compared with cancers without (8.4 vs $10.8 \mathrm{ng} /(\mathrm{dL} \times \mathrm{mL})$; odds ratio, OR 0.931; 95\% CI 0.873-0.994; $p=0.031$ ); so far, as ETD decreased, the risk of detecting PLNI increased, accordingly. Figure 2 depicts ROC curves of significant clinical continuous variables associated with the risk of PLNI; as illustrated, the association was positive for PSA and BPC, but inverse for ETD. So far, the risk of PLNI increased as PSA and BPC increased as well as ETD decreased, accordingly. On multivariate analysis, PLNI was independently predicted 
Table 1 Demographics of 201 high risk prostate cancer patients treated with radical prostatectomy and extended pelvic lymph node dissection

\begin{tabular}{|c|c|c|}
\hline Continuous variables & Mean (SD) & Median (IQR) \\
\hline Age (years) & $65.9(6.1)$ & $67(61.7-71)$ \\
\hline Body mass index; BMI $\left(\mathrm{kg} / \mathrm{m}^{\wedge} 2\right)$ & $26.2(3.3)$ & $25.6(24-28.7)$ \\
\hline Endogenous testosterone; ET (ng/dL) & $450.4(132.4)$ & $445(364.1-537.2)$ \\
\hline ET density; ETD (ng/(dL x mL)) & $11.9(7.38)$ & $10(7.1-15.1)$ \\
\hline Prostate specific antigen; PSA (ng/mL) & $12.5(15.6)$ & $7.5(5.4-14.1)$ \\
\hline PSA density; PSAD (ng/(mL x mL)) & $0.29(0.33)$ & $0.19(0.12-0.34)$ \\
\hline Prostate volume; PV (mL) & $44.7(18.4)$ & $41(30-57)$ \\
\hline Percentage of biopsy positive cores; BPC (\%) & $48.9(26.8)$ & $50(26.5-70)$ \\
\hline BPC density; BPCD (\%/mL) & $1.2(0.9)$ & $1.1(0.5-1.7)$ \\
\hline Prostate weight; PW (grams; gr) & $57.6(20.2)$ & $54.5(44.3-70)$ \\
\hline Tumor load; TL (\%) & $29.8(21.8)$ & $25(15-40)$ \\
\hline Tumor load density; TLD (\%/gr) & $0.5(0.4)$ & $0.43(0.21-0.70)$ \\
\hline Number od dissected lymph nodes; LN (n) & $26(9.4)$ & $26(20-32)$ \\
\hline Categorical variables & Number $(\%)$ & \\
\hline \multicolumn{3}{|l|}{ ISUP at biopsy } \\
\hline ISUP 1 & $25(12.4)$ & \\
\hline ISUP 2 & $36(17.9)$ & \\
\hline ISUP 3 & $26(12.9)$ & \\
\hline ISUP 4 & $93(46.3)$ & \\
\hline ISUP 5 & $21(10.5)$ & \\
\hline \multicolumn{3}{|l|}{ Clinical T stage (cT) } \\
\hline cT1c & $90(44.8)$ & \\
\hline $\mathrm{cT}>1$ & $111(55.2)$ & \\
\hline \multicolumn{3}{|l|}{ Clinical nodal stage $(\mathrm{cN})$} \\
\hline $\mathrm{cNO}$ & $167(83.1)$ & \\
\hline $\mathrm{cN} 1$ & $34(16.9)$ & \\
\hline \multicolumn{3}{|l|}{ ISUP at pathology } \\
\hline ISUP $=1$ & $8(4)$ & \\
\hline ISUP $=2$ & $28(13.9)$ & \\
\hline $\mathrm{ISUP}=3$ & $50(24.9)$ & \\
\hline ISUP $=4$ & $66(32.8)$ & \\
\hline $\mathrm{ISUP}=5$ & $49(24.4)$ & \\
\hline \multicolumn{3}{|l|}{ Pathologic tumor stage (pT) } \\
\hline pT2 & $120(62.7)$ & \\
\hline pT3a & $51(25.4)$ & \\
\hline pT3b & $24(11.9)$ & \\
\hline \multicolumn{3}{|l|}{ Surgical margins status (SM) } \\
\hline Negative (NSM) & $126(62.7)$ & \\
\hline Positive (PSM) & $75(37.3)$ & \\
\hline \multicolumn{3}{|l|}{ Pathologic nodal stage $(\mathrm{pN})$} \\
\hline pNO & $159(79.1)$ & \\
\hline $\mathrm{pN} 1$ & $42(20.9)$ & \\
\hline
\end{tabular}

only by BPC (OR 1.020; 95\% CI 1.006-1.035; $p=0.019$ ), ISUP $>3$ (OR 2.621; 95\% CI 1.170-5.869; $p=0.019)$ and ETD (OR 0.932; 95\% CI 0.870-0.999; $p=0.045$ ). Considering pathological parameters, PLNI was independently predicted by ISUP $>3$, seminal vesicle invasion, positive surgical margins and number of dissected lymph nodes, as detailed in Table 2. Continuous clinical predictors of PLNI were then categorized as follows: a BPC $>50 \%$ vs $<50 \%$; b ETD up to the median $(10 \mathrm{ng} /(\mathrm{mL} \times \mathrm{mL}))$ versus above. Table 3 describes analysis of clinical factors associated with the risk of PLNI. On univariate analysis, all categorized factors were positive predictors of PLNI. On multivariate analysis, the risk of PLNI was independently 
Table 2 Factors predicting pelvic lymph node invasion (PLNI) in 201 high risk prostate cancer patients treated with radical prostatectomy and extended pelvic lymph node dissection

\begin{tabular}{|c|c|c|c|c|c|c|}
\hline \multirow[t]{2}{*}{ Statistics } & \multirow{2}{*}{$\begin{array}{l}\text { No PLNI } \\
\text { Median (IQR) or } \\
\text { frequency (\%) }\end{array}$} & \multirow{2}{*}{$\begin{array}{l}\text { PLNI } \\
\text { Median (IQR) or frequency }(\%)\end{array}$} & \multicolumn{2}{|l|}{ Univariate analysis } & \multicolumn{2}{|l|}{ Multivariate analysis } \\
\hline & & & OR $(95 \% \mathrm{CI})$ & $\mathrm{P}$ value & OR $(95 \% \mathrm{CI})$ & $P$ value \\
\hline \multicolumn{7}{|c|}{ Clinical model } \\
\hline Age & $67(61-71)$ & $68.5(62.7-71)$ & $1.036(0.978-1.0 .98)$ & 0.232 & & \\
\hline BMI & $25.4(23.7-28.4)$ & $26.1(24.2-28.7)$ & $1.024(0.945-1.110)$ & 0.560 & & \\
\hline ET & $446.6(343-548)$ & $435.4(361.8-493.5)$ & $0.999(0.997-1.001)$ & 0.417 & & \\
\hline ETD & $10.8(7.2-16.3)$ & $8.4(6.7-11.7)$ & $0.931(0.873-0.994)$ & 0.031 & $0.932(0.870-0.999)$ & 0.045 \\
\hline PSA & $7.3(5.3-12.3)$ & $9.8(5.8-18.7)$ & $1.029(1.006-1.053)$ & 0.015 & $1.023(0.996-1.050)$ & 0.092 \\
\hline PSAD & $0.19(0.11-0.34)$ & $0.25(0.12-0.34)$ & $1.940(0.784-4.802)$ & 0.152 & & \\
\hline PV & $40(30-56)$ & $46.5(38-62.4)$ & $1.018(1.000-1.036)$ & 0.050 & & \\
\hline $\mathrm{BPC}$ & $38(25-60)$ & $58(38-50)$ & $1.023(1.010-1.037)$ & 0.001 & $1.020(1.006-1.035)$ & 0.006 \\
\hline BPCD & $1.00(0.48-1.66)$ & $1.23(0.62-1.71)$ & $1.109(0.799-1.538)$ & 0.537 & & \\
\hline ISUP $<4$ & $75(47.2)$ & $12(28.6)$ & Reference & & & \\
\hline ISUP $>3$ & $84(52.8)$ & $30(71.4)$ & $2.232(1.067-4.671)$ & $\mathbf{0 . 0 3 3}$ & $2.621(1.170-5.869)$ & 0.019 \\
\hline $\mathrm{cT} 1 \mathrm{c}$ & $77(44.8)$ & $13(31)$ & Reference & & & \\
\hline $\mathrm{cT}>1 \mathrm{c}$ & 82 (51.6) & $29(69)$ & $2.095(1.015-4.322)$ & 0.045 & $1.713(0.787-3.276)$ & 0.175 \\
\hline $\mathrm{cNO}$ & $130(81.8)$ & $37(88.1)$ & Reference & & & \\
\hline $\mathrm{cN} 1$ & $28(18.2)$ & $5(11.9)$ & $0.606(0.219-1.675)$ & 0.334 & & \\
\hline No RARP & $24(15.1)$ & $6(14.3)$ & Reference & & & \\
\hline RARP & $135(84.9)$ & $36(85.4)$ & $1.067(0.405-2.806)$ & 0.896 & & \\
\hline \multicolumn{7}{|c|}{ Pathological model } \\
\hline PW & $52(42-67)$ & $56(46.5-73.2)$ & $1.012(0.996-1.028)$ & 0.132 & & \\
\hline TLD & $0.36(0.18-0.66)$ & $0.62(0.44-1.03)$ & $3.178(1.582-6.387)$ & 0.001 & $1.817(0.799-4.135)$ & 0.155 \\
\hline ISUP $<4$ & $83(52.2)$ & $3(7.1)$ & Reference & & & \\
\hline ISUP $>3$ & $76(47.8)$ & 39 (92.9) & $14.197(4.213-47.841)$ & $<0.0001$ & $9.969(2.779-35.768)$ & $<0.0001$ \\
\hline $\mathrm{pT} 2$ & $106(66.7)$ & $14(33.3)$ & Reference & & & \\
\hline pT3a & $25(15.7)$ & $3(7.1)$ & $0.909(0.242-3.404)$ & 0.887 & & \\
\hline pT3b & $28(17.6)$ & $25(59.5)$ & $6.760(3.113-14.682)$ & $<0.0001$ & $3.509(1.564-8.188)$ & 0.004 \\
\hline NSM & $10(69.2)$ & $16(38.1)$ & Reference & & & \\
\hline PSM & $49(30.8)$ & $26(61.9)$ & 3.648 (1.797-7.405) & $<0.0001$ & $2.713(1.147-6.421)$ & 0.023 \\
\hline $\mathrm{LN}(\mathrm{n})$ & $25(19-31)$ & $29(23-34.7)$ & $1.049(1.013-1.086)$ & 0.007 & $1.096(1.017-1.180)$ & 0.016 \\
\hline
\end{tabular}

$I Q R$ interquartile range; $O R$ odds ratio; $C I$ confidence interval; see also Table 1

Factors predicting PLNI for which a statistically significant associations was found in the univariate and multivariate analysis are reported in bold

increased by ETD up to the median (OR 2.379; $95 \% \mathrm{CI}$ 1.134-4.991; $p=0.022), \mathrm{BPC}>50 \%(\mathrm{OR} 3.125 ; 95 \% \mathrm{CI}$ $1.520-6.425 ; p=0.002)$ as well as by ISUP > 3 (OR 2.219; $95 \%$ CI $1.031-4.776 ; p=0.042)$. As detailed in Table 3 and illustrated in Fig. 3, patients presenting with ETD up the median were more likely to occult PLNI $(27.7 \%)$ compared with those with ETD above the median (14\%). ETD was also tested on a multivariate model including significant pathological predictors of PLNI, as reported in supplementary Table S2. After adjusting for ISUP $>3$, seminal vesicle invasion, positive surgical margins and number of counted lymph nodes, ETD up to the median was still an independent predictor of the risk of PLNI (OR 2.592; 95\% CI 1.071-6.275; $p=0.035)$. Further details are described in Table $\mathrm{S} 2$.

\section{Discussion}

Treatment of HR-PCa with RP and ePLND is actually increasing; however, uniformity in definitions and indications, which impact on oncological outcomes, are missing [12, 13]. Moreover, when comparing surgery versus 


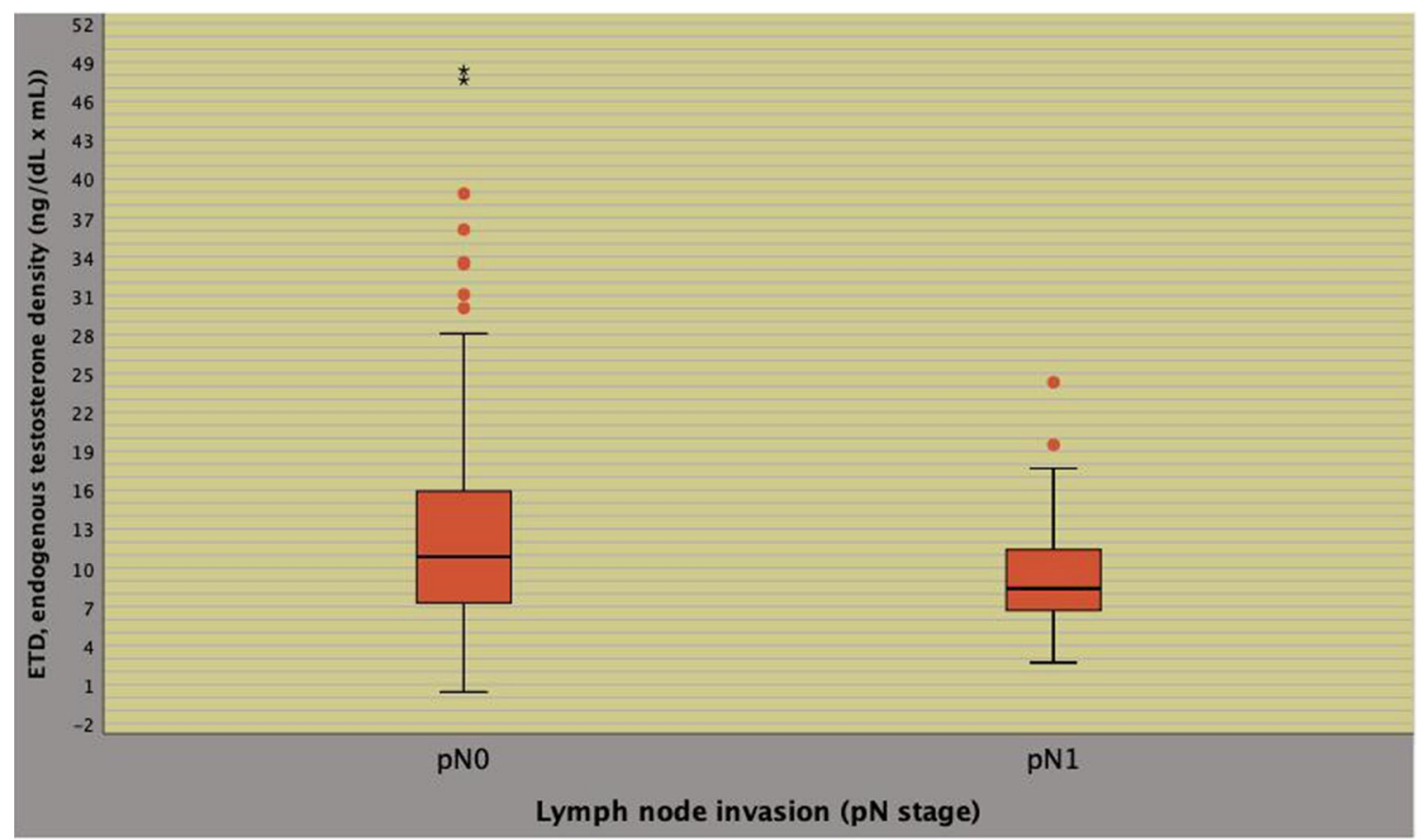

Fig. 1 Inverse association between endogenous testosterone density (ETD) and risk of pelvic lymph node invasion (PLNI). Significantly lower median levels of ETD were detected in tumors associated with
PLNI compared with cancers without (8.4 vs $10.8 \mathrm{ng} /(\mathrm{dL} \times \mathrm{mL})$; odd ratio, OR 0.931; 95\% CI 0.873-0.994; $p=0.031)$. As ETD decreased, the risk of detecting PLNI increased, accordingly

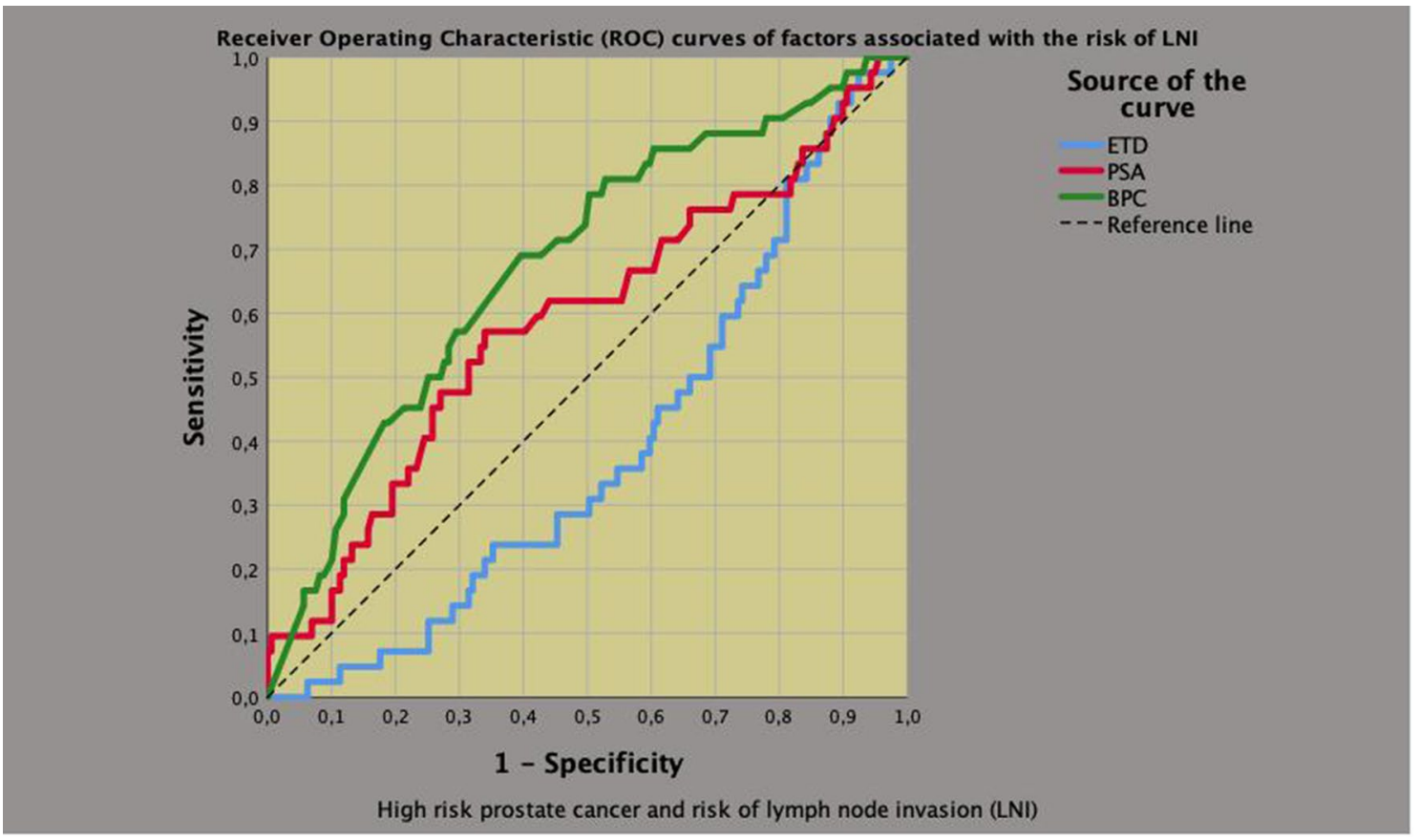

Fig. 2 Receiver operating characteristic (ROC) curves of significant clinical continuous variables associated with the risk of pelvic lymph node invasion (PLNI), which was positive for prostate specific antigen (PSA) and percentage of biopsy positive cores (BPC), but inverse for endogenous testosterone density (ETD). The risk of
PLNI increased as PSA and BPC increased as well as ETD decreased, accordingly. Area under the curves (AUC) and (95\% CI) were as follows: a ETD: $0.389(0.300-0.477 ; p=0.026)$; b BPC: 0.678 (0.588$0.769 ; p<0.0001)$; c PSA: $0.588(0.486-0.690 ; p=0.079)$ 
Table 3 Clinical factors associated with the risk of pelvic lymph node invasion in 201 high risk prostate cancer patients

\begin{tabular}{|c|c|c|c|c|c|c|c|}
\hline \multirow[t]{2}{*}{ Statistics } & \multirow[t]{2}{*}{$n(\%)$} & \multirow{2}{*}{$\begin{array}{l}\text { No PLNI } \\
n(\%)\end{array}$} & \multirow{2}{*}{$\begin{array}{l}\text { PLNI } \\
n(\%)\end{array}$} & \multicolumn{2}{|l|}{ Univariate analysis } & \multicolumn{2}{|l|}{ Multivariate analysis } \\
\hline & & & & OR $(95 \% \mathrm{CI})$ & $p$ value & OR $(95 \%$ CI $)$ & $p$ value \\
\hline \multicolumn{8}{|l|}{ ETD } \\
\hline Above the median & $100(49.8)$ & $86(86)$ & $14(14)$ & 1 & & 1 & \\
\hline Up to the median & $101(50.2)$ & $73(72.3)$ & $28(27.7)$ & 2356 (1155-4809) & 0.019 & $2379(1134-4991)$ & 0.022 \\
\hline \multicolumn{8}{|l|}{$\mathrm{BPC}$} \\
\hline Up to $50 \%$ & $128(63.7)$ & $110(85.9)$ & $18(14.1)$ & 1 & & 1 & \\
\hline Above $50 \%$ & $73(36.3)$ & $49(67.1)$ & $24(32.9)$ & $2993(1490-6014)$ & 0.002 & $3125(1520-6425)$ & 0.002 \\
\hline \multicolumn{8}{|l|}{ ISUP (biopsy) } \\
\hline Up to 3 & $87(43.3)$ & $75(86.2)$ & $12(13.8)$ & 1 & & 1 & \\
\hline Above 3 & $114(56.7)$ & $84(73.7)$ & $30(26.3)$ & $2232(1067-4671)$ & 0.033 & $2219(1031-4776)$ & 0.042 \\
\hline
\end{tabular}

$P L N I$ pelvic lymph node invasion; $O R$ odd ratio; $C I$ confidence interval; $E D T$ endogenous testosterone density; $B P C$ percentage of biopsy positive cores; ISUP International Society of Urologic Pathology tumor grade system; see also results for further details

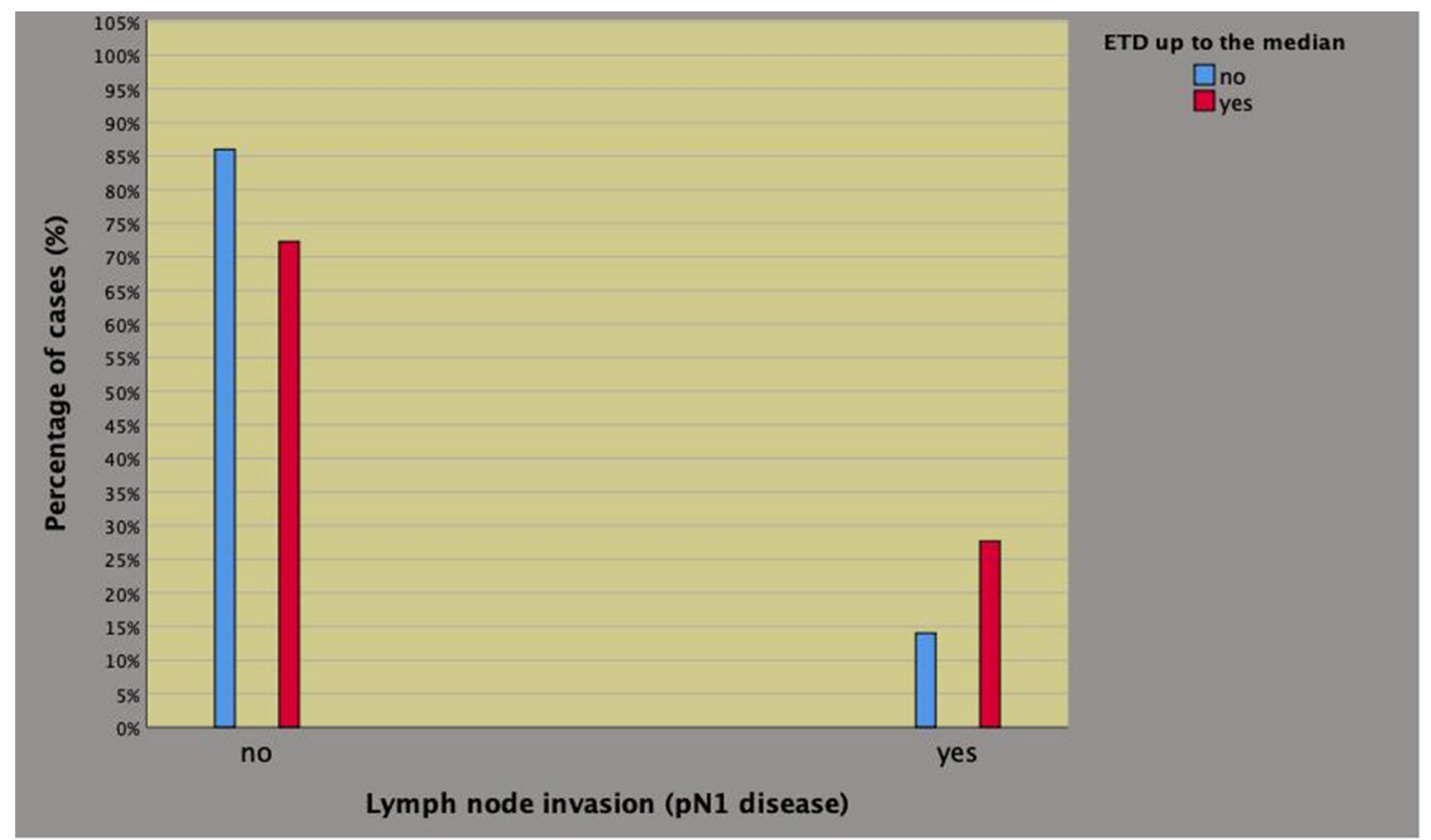

Fig. 3 Endogenous testosterone density (ETD) associated with the risk of pelvic lymph node invasion (PLNI) in patients with high-risk prostate cancer treated with radical prostatectomy and extended pelvic lymph node dissection. Patients presenting with ETD up to the median were more likely to occult PLNI (27.7\%) compared with subjects having ETD measurements above the median (14\%) and the

radiation and androgen deprivation therapy as primary treatments of HR-PCa, evidence showing definitive superiority of either modality is lacking [13]. In clinical practice, a critical issue is assessment of PLNI. Although several validated nomograms, which refer to commonly available clinical factors, have been proposed, there is a lack of specificity for HR-PCa [1, 2]. Recently, new nomograms have been validated through mpMRI parameters including the PI-RADS association was significant on both univariate analysis (OR 2.356; 95\% CI $1.155-4.809 ; p=0.019)$ as well as after adjusting for percentage of biopsy positive cores above 50\% and tumor grade above 3 according to the International Society of Urologic Pathology (ISUP) system (adjusted OR 2.379; 95\% CI 1.134-4.991; $p=0.022$ ). See also Table 3 and results for further details

score, number of PI-RADS $\geq 3$ lesions, maximum diameter of index lesion and tumor stage (organ confined, extracapsular extension and seminal vesicle invasion), as well [14]. A European multicenter study while externally validating the currently available nomograms in predicting PLNI, found out that the MSKCC and Briganti 2012 nomograms were superior in predicting nodal metastases suggesting that mpMRI parameters did not add any relevant information for 
not being reproducible in the different centers because of the high dependency on the operator [15].So far, further simpler and newer parameters are required to validate future nomograms predicting the risk of PLNI [16]. Our study showed that ETD either a continuous or categorized variable could be an effective biomarker for assessing occult PLNI in HR$\mathrm{PCa}$ according to the EAU system, independently from other clinical or pathological because of the strong association with tumor aggressive biology; however, confirmatory studies are required.

In locally advanced disease, the triangle of Marcille's as well as Cloquet's node are vital anatomical regions for advanced pelvic surgery in evaluating systemic nodal disease, as well $[17,18]$. These findings explain why pN1 high risk patients with biochemical persistence/recurrence have their first detectable recurrence on gallium- 68 prostatespecific membrane antigen positron-emission tomography (PET)/computed tomography (CT) in the prostatic fossa and/ or pelvic nodes (2/3 of positive cases) as well as outside the pelvic area in distant (retroperitoneal) lymph nodes and/or bones (1/3 of cases), as well $[19,20]$. So far, predictors of advanced pelvic disease are actually required in HR-PCa. Our study has specifically investigated the issue of PLNI in HR disease, which was detected in $20.9 \%$ of cases; furthermore, anatomical staging was appropriate for either extension of the template as well as for the median (IQR) number of removed nodes, which was 26 (20-32). As such, the predictivity of ETD was tested in a population who was extensively staged anatomically according to an advanced pelvic pattern including extremal territories of prostatic lymphatic system represented by Marcille's fossa and Cloquet's nodes, which are pivotal landmarks for evaluating PLNI in advanced disease $[9,10,17,18]$. However, comparative studies for evaluating this issue are actually missing.

Although PCa is a hormone dependent tumor, the subject dealing with associations between ET levels and aggressive cancer features is highly controversial for controlled studies are missing and for ET not being measured on a chronic basis, as it should be [21, 22]. According to systematic reviews and meta-analysis, the association may be found positive, inverse or null [21, 22]. However, when considering associations of ET with PLNI, few studies are available; specifically, they show significant inverse associations between ET levels and risk of PLNI. Recently, we have confirmed these results showing that, in early PCa undergoing ePLND and including all risk classes according to the EAU system, there is an inverse association between ET and risk of multiple occultic metastases at loco-regional lymph nodes [23]. In the present study, we have demonstrated that ETD was an independent parameter for predicting occult metastatic disease in the high-risk population in whom as ETD decreased, the risk of PLNI increased accordingly, independently by load (BPC $>50 \%$ ) and/or grade (ISUP $>3$ ) of the tumor, as well. Although adjusting ET for prostate volumes is a novelty for HR disease, we have already investigated this feature in the low-risk category. Specifically, we have shown that ETD associated with the risk of high tumor load (percentage of cancer involving at least $20 \%$ of the gland) that predicted the risk of unfavorable disease; furthermore, although that study is not comparable with the present one for several features, either investigation showed associations of ETD with factors related to aggressive PCa, as well [7]. The results of the present study represent a novelty for the literature dealing with this topic and might have implications in clinical practice.

In HR disease with biopsy ISUP $>3$, the incidence of downgrading ranges between 26 and $31 \%$; as such, patients presenting with low ETD (up to the median) will have an increased risk of PLNI, which might be an important issue to discuss when counselling patients. Particularly, these patients should not be candidate to focal therapy [24, 25] as already suggested by international guidelines [1, 2]. Additionally, in HR locally advanced PCa treated with RP and ePLND, the risk of PLNI may be evaluated by EDT together with other routinely factors. Furthermore, $\mathrm{pN} 1$ patients with biochemical persistence/recurrence with low ETD measurements might occult residual disease in the pelvic and extrapelvic nodes, independently by PET-CT PSMA results [1, $2,19]$. All these implications suggest the need of prospective controlled trials in order to assess the influence of $\mathrm{PCa}$ biology on either ET and ETD in the HR category, as well.

Our study has several limitations. Prostate volumes were not all measured at our institution. ET was measured only once and not on a chronic base, as recommended [22]. Central pathology review of external biopsies was not performed. Results of mpMRI were not evaluated for not being available in all patients. Genetic tests were not performed. Analysis of maximal cancer involvement of each core was not computed for not being available in all patients. Finally, the retrospective nature of the study. Our study has strengths, as well. All prostate specimens were assessed by our dedicated pathologist. ET was measured in the morning, which is the appropriate interval for evaluating the levels of the hormone, which decrease in the afternoon, as well [26]. Data were prospectively collected. It was single center study, and the patient population was homogenous for ethnicity (Caucasian) and for ET measurements, which were all performed at our laboratory.

\section{Conclusions}

ETD, which was positively correlated to ET, inversely associated with the risk of PLNI; as ETD decreased, the risk of PLNI increased, accordingly. The influence of HR disease on 
either ET levels and ETD measurements needs to be evaluated by prospective multicenter studies.

Supplementary Information The online version contains supplementary material available at https://doi.org/10.1007/s11255-022-03103-w.

Funding The authors didn't receive financial support.

\section{Declarations}

Conflict of interest The authors declare that they have no conflict of interest.

Ethical approval All procedures performed in studies involving human participants were in accordance with the ethical standards of the institutional and/or national research committee and with the 1964 Helsinki declaration and its later amendments or comparable ethical standards.

Informed consent Informed consent was obtained from all individual participants included in the study.

Open Access This article is licensed under a Creative Commons Attribution 4.0 International License, which permits use, sharing, adaptation, distribution and reproduction in any medium or format, as long as you give appropriate credit to the original author(s) and the source, provide a link to the Creative Commons licence, and indicate if changes were made. The images or other third party material in this article are included in the article's Creative Commons licence, unless indicated otherwise in a credit line to the material. If material is not included in the article's Creative Commons licence and your intended use is not permitted by statutory regulation or exceeds the permitted use, you will need to obtain permission directly from the copyright holder. To view a copy of this licence, visit http://creativecommons.org/licenses/by/4.0/.

\section{References}

1. Mottet N, Bellmunt J, Bolla M et al (2017) EAU-ESTRO-SIOG guidelines on prostate cancer. Part 1: screening, diagnosis, and local treatment with curative intent. Eur Urol 71(4):618-629

2. Mohler JL, Antonarakis ES, Armstrong AJ et al (2019) Prostate cancer, version 2.2019, NCCN clinical practice guidelines in oncology. J Nat Comprehens Cancer Netw 17(5):479-505

3. Artibani W, Porcaro AB, De Marco V et al (2018) Management of biochemical recurrence after primary curative treatment for prostate cancer: a review. Urol Int 100(3):251-262

4. Porcaro AB, Amigoni N, Tafuri A et al (2021) Endogenous testosterone as a predictor of prostate growing disorders in the aging male. Int Urol Nephrol 53(5):843-854

5. Tafuri A, Amigoni N, Rizzetto R et al (2020) Obesity strongly predicts clinically undetected multiple lymph node metastases in intermediate-and high-risk prostate cancer patients who underwent robot assisted radical prostatectomy and extended lymph node dissection. Int Urol Nephrol 52(11):2097-2105

6. Porcaro AB, Tafuri A, Sebben M et al (2020) High body mass index predicts multiple prostate cancer lymph node metastases after radical prostatectomy and extended pelvic lymph node dissection. Asian J Androl 22(3):323

7. Porcaro AB, Tafuri A, Sebben M et al (2019) Total testosterone density predicts high tumor load and disease reclassification of prostate cancer: results in 144 low-risk patients who underwent radical prostatectomy. Int Urol Nephrol 51(12):2169-2180
8. Porcaro AB, Tafuri A, Sebben M et al (2020) Prostate volume index and prostatic chronic inflammation predicted low tumor load in 945 patients at baseline prostate biopsy. World J Urol 38(4):957-964

9. Porcaro AB, Cacciamani GE, Sebben M et al (2019) Lymph nodes invasion of Marcille's fossa associates with high metastatic load in prostate cancer patients undergoing extended pelvic lymph node dissection: the role of "Marcillectomy." Urol Int 103(1):25-32

10. Cacciamani GE, Porcaro AB, Sebben M et al (2019) Extended pelvic lymphadenectomy for prostate cancer: should the Cloquet's nodes dissection be considered only an option? Minerva Urol Nefrol = Italian J Urol Nephrol 71(2):136-145

11. Van Der Kwast TH, Amin MB, Billis A et al (2011) International Society of Urological Pathology (ISUP) consensus conference on handling and staging of radical prostatectomy specimens. Working group 2: T2 substaging and prostate cancer volume. Mod Pathol 24(1):16-25

12. Delporte G, Henon F, Ploussard G et al (2018) Radical prostatectomy for locally advanced and high-risk prostate cancer: a systematic review of the literature. Prog Urol 28(16):875-889

13. Knipper S, Karakiewicz PI, Heinze A et al (2020) Definition of high-risk prostate cancer impacts oncological outcomes after radical prostatectomy. Urol Oncol Semin Original Investig. https://doi. org/10.1016/j.urolonc.2019.12.014

14. Gandaglia G, Ploussard G, Valerio M et al (2019) A novel nomogram to identify candidates for extended pelvic lymph node dissection among patients with clinically localized prostate cancer diagnosed with magnetic resonance imaging-targeted and systematic biopsies. Eur Urol 75(3):506-514

15. Oderda M, Diamand R, Albisinni S et al (2021) Indications for and complications of pelvic lymph node dissection in prostate cancer: accuracy of available nomograms for the prediction of lymph node invasion. BJU Int 127(3):318-325

16. McKay RR, Feng FY, Wang AY et al (2020) Recent advances in the management of high-risk localized prostate cancer: local therapy, systemic therapy, and biomarkers to guide treatment decisions. Am Soc Clin Oncol Educ Book 40:e241-e252

17. Maderthaner L, Furrer MA, Studer UE et al (2018) More extended lymph node dissection template at radical prostatectomy detects metastases in the common iliac region and in the fossa of Marcille. BJU Int 121(5):725-731

18. Sebben M, Tafuri A, Porcaro AB et al (2020) Response to: Bando et al. Diagnostic and therapeutic value of pelvic lymph node dissection in the fossa of Marcille in patients with clinically localized high-risk prostate cancer Histological and molecular analyses. Prostate 80(10):795-796

19. Huits TH, Luiting HB, van Der Poel HG et al (2020) Distribution of prostate cancer recurrences on gallium- 68 prostate-specific membrane antigen (68Ga-PSMA) positron-emission/computed tomography after radical prostatectomy with pathological nodepositive extended lymph node dissection. BJU Int 125(6):876

20. Porcaro AB, De Luyk N, Corsi P et al (2017) Clinical factors predicting bilateral lymph node invasion in high-risk prostate cancer. Urol Int 99(4):392-399

21. Klap J, Schmid M, Loughlin KR (2015) The relationship between total testosterone levels and prostate cancer: a review of the continuing controversy. J Urol 193(2):403-414

22. Loughlin KR (2016) The testosterone conundrum: the putative relationship between testosterone levels and prostate cancer. Urol Oncol Semin Orig Investig. https://doi.org/10.1016/j.urolonc. 2016.05.023

23. Porcaro AB, Cerrato C, Tafuri A et al (2021) Low endogenous testosterone levels are associated with the extend of lymphnodal invasion at radical prostatectomy and extended pelvic lymph node dissection. Int Urol Nephrol 53(10):2027-2039 
24. Oishi M, Gill IS, Tafuri A et al (2019) Hemigland cryoablation of localized low, intermediate and high risk prostate cancer: oncologic and functional outcomes at 5 years. J Urol 202(6):1188-1197

25. Abreu AL, Peretsman S, Iwata A et al (2020) High intensity focused ultrasound hemigland ablation for prostate cancer: initial outcomes of a United States series. J Urol 204(4):741-747

26. Brambilla DJ, Matsumoto AM, Araujo AB et al (2009) The effect of diurnal variation on clinical measurement of serum testosterone and other sex hormone levels in men. J Clin Endocrinol Metab 94(3):907-913

Publisher's Note Springer Nature remains neutral with regard to jurisdictional claims in published maps and institutional affiliations. 